BMJ Paediatrics Open

\title{
Systematic review of mydriatics used for screening of retinopathy in premature infants
}

Lisa Jean Kremer, ${ }^{\oplus 1,2}$ David M Reith, ${ }^{2}$ Natalie Medlicott, ${ }^{1}$ Roland Broadbent ${ }^{2}$

\section{ABSTRACT}

Introduction Routine retinopathy of prematurity eye examinations are an important part of neonatal care, and mydriatic medicines are essential in dilating the pupil for the eye examination. There are concerns about the level of evidence for efficacy and safety of these mydriatic medicines.

Objective This review evaluates both efficacy and safety evidence of mydriatics used during the retinopathy of prematurity eye examination.

Method Systematic literature review.

Results There is limited evidence guiding clinical practice for safety and efficacy of mydriatics. The majority of publications are underpowered and with an unclear to high level of bias. There are a wide variety of mydriatic regimens evaluated for efficacy and safety, and multiple regimens are associated with case reports.

Conclusions Current international guideline seems unnecessarily high, especially when the reviewed literature suggest that lower doses are effective, albiet from underpowered studies. The lowest effective combination regimen appears to be phenylephrine $1 \%$ and cyclopentolate $0.2 \%$ (1 drop). Microdrop administration of this regimen would further increase the safety profile, however, efficacy needs to be assessed.

\section{INTRODUCTION}

Retinopathy is a common complication of prematurity, and routine screening examinations are essential for timely diagnosis of retinopathy of prematurity (ROP). It is essential to have timely diagnosis and treatment of ROP, to prevent permanent blindness in premature neonates; however, it is essential that we have both safe and effective mydriatic regimens.

Mydriatic medicines play an essential diagnostic role; however, there are clinically significant risks associated with using mydriatics. There are concerns about the limited evidence that is used to support safe and effective mydriatic regimens, for retinopathy of prematurity eye examinations (ROPEE).

Various combinations of phenylephrine, cyclopentolate and tropicamide are commonly used to prepare the neonatal for the ROPEE. However, the lowest effective dose has not been substantiated with a large data set nor has the evaluation of microdrop (md) to standard drop (d) administration. Pupil dilation results from standard and microdrop size was included in the analysis, and for the purposes of this paper, a standard drop size is considered to be approximately $26 \mu \mathrm{L}$, and a microdrop size is approximately $7 \mu \mathrm{L}^{1}$

The main safety concerns surrounding mydriatic medicines are ones associated with systemic absorption following ocular administration. Following ocular delivery, it is estimated that approximately $80 \%$ of the eye drop volume enters the nasolacrimal duct, therefore gaining entry into the systemic circulation via the nasal mucosa. ${ }^{2}$ Systemic absorption of mydriatics have been associated with clinically significant, and sometimes life-threatening cardiovascular, respiratory, central nervous system (CNS) and gastrointestinal adverse effects. Examples of some adverse effects include: hypertension, hypotension, tachycardia, bradycardia, apnoea, cardiopulmonary arrest, seizures, necrotising enterocolitis (NEC), sepsis and death.

Management of these side effects are challenging and premature infants have less ability to cope with the associated problems. Management of side effects in premature infants born in the developing world is of great concern as they are likely to have reduced access to care and management of the associated complications.

International guidelines play an essential role in directing best practice worldwide. The two readily available international guidelines for ROPEE are the UK Guideline for the Screening and Treatment of Retinopathy of Prematurity, 2008 and the American Policy Statement on Screening Examination of Premature Infants for Retinopathy of Prematurity, 2013. ${ }^{34}$

The UK guideline recommends phenylephrine $2.5 \%$ and cyclopentolate $0.5 \%, 2-3 \mathrm{~d}$; however, a lower dose regimen is favourable 
but not recommended because a proprietary product is not available. In comparison, the American policy statement does not provide any recommendations for suitable mydriatic agents and regimens.

This robust review of the literature evaluates the evidence for both the safety and efficacy aspects of mydriatic regimens used during ROP screening.

\section{METHODS}

One review author (LK) searched the published literature looking for reports on the safety and/or efficacy during 2017 and 2018.

\section{Databases used}

The following databases were used: EMBASE, MEDLINE via Ovid, PubMed, Web of Science, Cochrane Library and Cochrane Neonatal Review Group and Google Scholar. Date restricted 1970 onwards. Refer to online appendix 1 for Medline search strategy.

\section{Other}

Additional records were identified through reference review of sourced literature.

\section{Keywords used}

The following keywords were used 'phenylephrine', 'cyclopentolate', 'tropicamide', 'mydriasis', 'pupil dilation', 'adverse effects', 'administration and dosage', 'eye drops', 'ophthalmic solutions', 'pupil'. Subject heading: 'infant, newborn, premature', 'efficacy', 'safety'. It was considered that the following were synonymous with mydriasis: pupil dilation and pupil dilatation.

\section{Inclusion criteria}

Participants are premature infants, interventions are mydriatic medicine administration, comparisons of efficacy are between different mydriatic regimens, comparisons of safety are between baseline and any adverse effect, outcomes for efficacy are either pupil dilation measurements or successful eye examination, outcomes for safety are any adverse effects, study design includes all study designs including pilot and case reports. Full text is available in English.

\section{Exclusion criteria}

Full text not available in English, published after 1970, commentary, letters to the editor, publications on; pain associated with ROP procedure, physiology and ophthalmic formulation; paediatric participants.

\section{Study selection}

Screening and eligibility assessment was performed independently in an unblinded standardised manner by one reviewer (LK). No data was meta-analysed due to the high variability of the mydriatic regimens.

\section{Data collection process}

Included studies were entered into EndNote, and the 'remove duplicates' function was utilised. Online trial registration for all intervention studies was accessed (when available) and study design was compared with publication results.

Studies that reported pupil dilation data were entered into Microsoft Excel and a forest plot was created using GraphPad PRISM (V.7.04). Some of the pupil dilation data needed to be converted into measurement $(\mathrm{mm})$ and rounded to the nearest decimal point. Studies that reported blood pressure and heart rate data were collated in a Microsoft Word table. Some of the blood pressure results were converted into percentage increase or decrease.

\section{Data items}

Information was extracted from each included trial on: (1) characteristics of trial participants (including gestational age, birth weight) and the trial's inclusion and exclusion criteria; (2) type of intervention (mydriatic(s) used, concentration, number of administrations, timing of dosing; (3) type of outcome measure (including pupil dilation measurement, effects on cardiovascular, gastrointestinal, respiratory, dermal and CNS and other).

\section{Risk of bias}

Cochrane methodology was used to assess risk of bias for RCTs and results analysed using Review Manager (V.5.3). Risk of bias in non-randomised studies - of interventions (ROBINS-I) tool was used to assess risk of bias for non-randomised intervention trials, and observational studies were assessed for bias using the National Heart, Lung and Blood Institute (NHLBI) checklist. ${ }^{5}$

\section{Summary measures}

Where available, the difference in means $( \pm \mathrm{SD})$ data was obtained from the pupil dilation (efficacy) and safety measurements (eg, blood pressure, heart rate).

\section{RESULTS}

One hundred and eighty six studies were identified, and of these, 47 publications were included in the systematic review (figure 1). Efficacy and safety data were extracted from the publications, and safety data were further subcategorised into themes of cardiovascular, gastrointestinal, respiratory, CNS, dermal and other effects (table 1). All trials occurred at a single centre.

Risk of bias for all studies are summarised in figure 2 and in tables 2 and 3 .

\section{Efficacy}

Key findings

1. The majority of published data are from pilot studies that had a significant amount of unclear to high risk of bias; therefore, there is no strong evidence to support clinical practice (figure 2). 


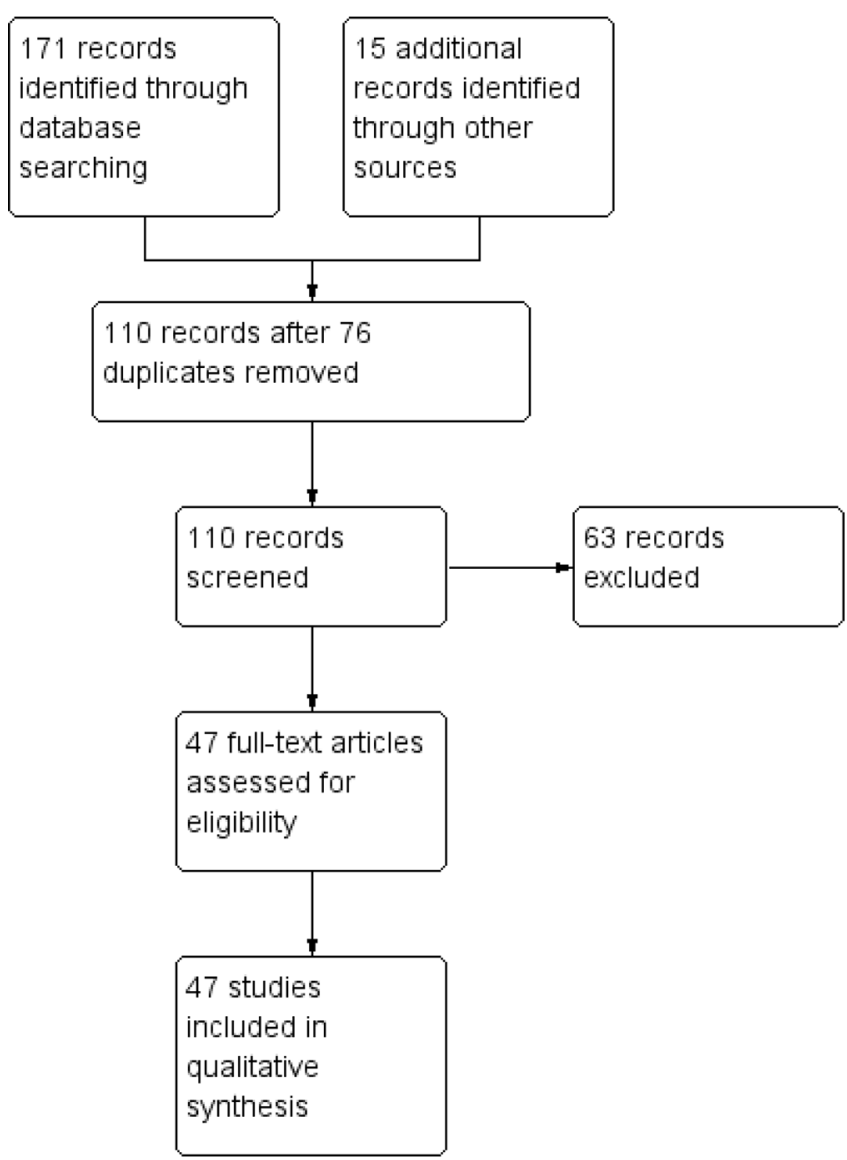

Figure 1 Preferred Reporting Items for Systematic Reviews and Meta-Analyses flow diagram.

2. There is a wide range of mydriatic concentrations and frequency of administration in the published literature.

3. Almost all mydriatic regimens included in this review demonstrated sufficient pupil dilation of above $5 \mathrm{~mm}$ (figure 3).

4. The lowest effective regimen appears to be phenylephrine $1 \%$ and cyclopentolate $0.2 \%, 1 \mathrm{~d}$ of each.

5. Microdrop administration of mydriatic medicines are likely to sufficiently dilate the pupil.

Fifteen studies measured pupil dilation to ascertain efficacy and one publication (Neffendorf 2014) measured efficacy as a successful ROPEE. Participants were all premature infants and the number of participants in the studies ranged from 5 to 138. Study design was either RCT, quasi-RCT, cross-over controlled RCT, or an audit; however, the majority of the studies were quasi-RCTs and the majority had small sample sizes. Within these studies, the mydriatic regimens, method of measuring pupil diameter, physiological measures (eg, blood pressure, feed intolerance) all varied significantly. Because of this diverse range of mydriatic regimens and study methodology, comparisons across papers posed some difficulty and therefore highlights the need for robust evidence to guide clinical practice.
Additionally, within these 16 studies, there were 33 different regimens using various combinations of phenylephrine, cyclopentolate and tropicamide (table 1).

Only three regimens did not achieve sufficient pupil dilation above $5 \mathrm{~mm}$ : monotherapy with phenylephrine $10 \%(2 \mathrm{~d} \text { and } 2 \text { microd })^{6}$ and tropicamide $0.5 \%(3 \mathrm{~d}){ }^{7}$ However, all other regimens, irrespective of concentration and frequency, did provide sufficient dilation (figure 3). Data from Isenberg (1984 March and July) is not included in figure 3 , because net pupil dilation results were not available. ${ }^{89}$ Data from Wheatcroft (1993) was also unable to be used in figure 3 because SD results were not available. ${ }^{1}$

Overall, the efficacy studies had a significant amount of unclear bias. Of concern, the majority of these studies had unclear randomisation generation, allocation concealment, blinding of personnel and participants (figure 2).

\section{Safety}

Key findings

1. The majority of safety specific data is from low level study design studies; therefore, there is limited evidence to support clinical practice (figure 2 and tables 2 and 3 ).

2. There is a diverse range of safety aspects evaluating mydriatic safety.

3. Multiple measures were used to evaluate mydriatic safety.

Twenty-two studies investigated safety aspects of the mydriatic regimens (table 1). Within case reports, there were 30 adverse events post-mydriatic administration.

Participants in the safety studies and case reports were all premature infants. The safety study design was either RCT, non-randomised intervention, observational, retrospective audits or case reports. The majority of the studies were non-randmoised intervention studies, and the sample size between the safety studies ranged from 7 to 1183 . Within these studies, the mydriatic regimens, physiological measures (eg, blood pressure, feed intolerance) all varied significantly. This was similar for the case report analysis. Comparisons across papers created difficulty because of the variation in study design, low level of study design and the wide range of mydriatic regimens.

\section{Cardiovascular}

Key findings

1. Systemic absorption of phenylephrine causes vasoconstriction, resulting in increased blood pressure and tachycardia, ${ }^{10-12}$ whereas anticholinergics can cause transient bradycardia, followed by tachycardia, palpitation and arrhythmias. ${ }^{13}$ Both increases and decreases in blood pressure and heart rate were reported in the literature.

2. There is a wide range of both mydriatic regimens and blood pressure measures, therefore, making the interpretation of cardiovascular effects challenging. 
Open access

Table 1 Summary of characteristics and demographics for publications (excluding case reports)

\begin{tabular}{|c|c|c|c|c|c|c|c|}
\hline Paper & Design & Regimen & Mean (SD) GA & Mean (SD) BW (g) & Sample Size & Efficacy & Safety \\
\hline Alshafei $(2017)^{6}$ & $\begin{array}{l}\text { Observational } \\
\text { prospective }\end{array}$ & P $2.5 \%$, T $5 \%$ (3 d) & $24-31$ & - & 42 & & $\mathrm{R}$ \\
\hline \multirow[t]{2}{*}{ Bolt $(1992)^{7}$} & \multirow[t]{2}{*}{ Quasi-RCT } & P $2.5 \%$ (1d), T $0.5 \%$ (2 d) & $32.3( \pm 3.3)$ weeks & $1544( \pm 761)$ & 20 & $\checkmark$ & $\mathrm{C}$ \\
\hline & & C $0.5 \%(1 d)$, T $0.5 \%(2 d)$ & $34.6( \pm 3.6)$ weeks & $1831( \pm 645)$ & 19 & $\checkmark$ & $\mathrm{C}$ \\
\hline Bonthala $(2000)^{8}$ & $\begin{array}{l}\text { Non-randomised } \\
\text { intervention }\end{array}$ & P $1 \%, C 0.2 \%(2 d)$ & $29-33$ & $1340( \pm 82)$ & 11 & & C \\
\hline Caputo $(1982)^{9}$ & Quasi-RCT & $\begin{array}{l}\text { P } 2.5 \%, \text { C } 0.5 \%, \text { T } 0.5 \% \\
\text { (2 d) }\end{array}$ & 26-42 weeks & $880-3440$ & 10 & $\checkmark$ & \\
\hline \multirow[t]{2}{*}{ Chew $(2005)^{20}$} & \multirow[t]{2}{*}{$\mathrm{RCT}$} & P $2.5 \%, C 1 \%(3 \mathrm{~d})$ & $\begin{array}{l}29.92( \pm 2.66) \\
\text { weeks }\end{array}$ & - & 13 & $\checkmark$ & G \\
\hline & & P 2.5\%, T 1\% (3 d) & $\begin{array}{l}29.23( \pm 1.59) \\
\text { weeks }\end{array}$ & - & 13 & $\checkmark$ & $\mathrm{G}$ \\
\hline \multirow{2}{*}{ Elibol $(1997)^{17}$} & \multirow{2}{*}{ Quasi-RCT } & P $10 \%$ (2 d or 2 microd) & $\begin{array}{l}22.83( \pm 21.39) \\
\text { days }\end{array}$ & - & 18 & $\checkmark$ & C, D \\
\hline & & T $0.5 \%$ (2 d or 2 microd) & $\begin{array}{l}28.78( \pm 26.89) \\
\text { days }\end{array}$ & - & 19 & $\checkmark$ & $C, D$ \\
\hline \multirow[t]{3}{*}{ Isenberg $(1985)^{15}$} & \multirow[t]{3}{*}{$\mathrm{RCT}$} & C $0.25 \%(2 d)$ & $31( \pm 2)$ weeks & $1233( \pm 390)$ & 6 & & $G$ \\
\hline & & C $0.5 \%(2 d)$ & $31( \pm 2)$ weeks & $1233( \pm 390)$ & 8 & & $G$ \\
\hline & & Saline $0.9 \%$ & $31( \pm 2)$ weeks & $1233( \pm 390)$ & 6 & & \\
\hline \multirow{2}{*}{$\begin{array}{l}\text { Isenberg }(1984 \\
\text { March })^{18}\end{array}$} & \multirow{2}{*}{$\begin{array}{l}\text { Non-randomised } \\
\text { intervention }\end{array}$} & C $0.5 \%(2 d)$ & - & $1198( \pm 220)$ & 10 & $\checkmark$ & $\mathrm{C}$ \\
\hline & & C $0.5 \%, T 0.5 \%(2 \mathrm{~d})$ & - & $1227( \pm 220)$ & 10 & $\checkmark$ & $\mathrm{C}$ \\
\hline \multirow[t]{2}{*}{ Khoo $(2000)^{16}$} & \multirow[t]{2}{*}{ RCT crossover } & P 1\%, C $0.2 \%$ (3 d) & $\begin{array}{l}26.2 \text { weeks } \\
\text { (test 1) } \\
35.8 \text { weeks } \\
\text { (test 2) }\end{array}$ & - & 28 & $\checkmark$ & C \\
\hline & & P 2.5\%, T 0.5\% (3 d) & $\begin{array}{l}26.2 \text { weeks } \\
\text { (test } 1 \text { ) } \\
36.1 \text { weeks } \\
\text { (test 2) }\end{array}$ & - & 28 & $\checkmark$ & C \\
\hline Laws $(1996)^{11}$ & $\begin{array}{l}\text { Observational } \\
\text { prospective }\end{array}$ & P $25 \%$ (2d), C $0.5 \%(4$ d) & $27.1( \pm 2.4)$ & $1003( \pm 332)$ & 56 & & $\mathrm{C}$ \\
\hline Lees $(1981)^{12}$ & $\begin{array}{l}\text { Observational } \\
\text { prospective }\end{array}$ & P 2.5\%, T 0.5\% (1 d) & $31( \pm 3.12)$ & $1450( \pm 464)$ & 7 & & C \\
\hline \multirow[t]{2}{*}{ Luo $(2014)^{21}$} & \multirow[t]{2}{*}{ RCT crossover } & P $0.5 \%, T 0.5 \%(3 \mathrm{~d})$ & 26-37 weeks & - & 88 & $\checkmark$ & \\
\hline & & $P 0.25 \%, T 0.25 \%$ (3 d) & 26-37 weeks & - & 88 & $\checkmark$ & \\
\hline \multirow[t]{2}{*}{ Lux $(2016)^{27}$} & \multirow[t]{2}{*}{$\mathrm{RCT}$} & T $0.5 \%$ (3 d) & - & - & 30 & $\checkmark$ & \\
\hline & & P $5 \%$ (1 d), T 0.5\% (2 d) & - & - & 30 & $\checkmark$ & \\
\hline \multirow[t]{2}{*}{ Merrit $(1981)^{45}$} & \multirow[t]{2}{*}{$\mathrm{RCT}$} & P 2.5\%, T 1\% (3 d) & $32( \pm 0.5)$ & $1569( \pm 98.7)$ & 52 & & $\mathrm{C}$ \\
\hline & & $\begin{array}{l}\text { P } 2.5 \%, \text { T } 0.5 \%, \text { C } 0.5 \% \\
\text { (3 d) }\end{array}$ & $30.2( \pm 0.4)$ & $1250.7( \pm 61.6)$ & 30 & & $\mathrm{C}$ \\
\hline Mirmanesh (1992) ${ }^{22}$ & $\begin{array}{l}\text { Non-randomised } \\
\text { Intervention }\end{array}$ & P $2.5 \%$ (3 d) & $27( \pm 2)$ & $840( \pm 200)$ & 21 & & $\mathrm{R}$ \\
\hline
\end{tabular}




\begin{tabular}{|c|c|c|c|c|c|c|c|}
\hline Paper & Design & Regimen & Mean (SD) GA & Mean (SD) BW (g) & Sample Size & Efficacy & Safety \\
\hline Mitchell $(2016)^{28}$ & $\mathrm{RCT}$ & P 1\%, C 0.2\% (3 d) & $28.5( \pm 2.8)$ & $1148( \pm 523)$ & 25 & & $\mathrm{G}, \mathrm{R}$ \\
\hline Mitchell $(2011)^{46}$ & $\begin{array}{l}\text { Observational } \\
\text { prospective }\end{array}$ & P 1\%, C $0.2 \%$ (3 d) & $28.24( \pm 2.62)$ & $1161( \pm 352)$ & 50 & & $\mathrm{R}, \mathrm{C}, \mathrm{G}, \mathrm{N}$ \\
\hline Neffendorf $(2015)^{43}$ & $\begin{array}{l}\text { Observational } \\
\text { retrospective }\end{array}$ & P 2.5\%, C 0.5\% (3 d) & 29 (no SD) & 1234 (no SD) & 138 & $\checkmark$ & $\mathrm{R}$, C, other \\
\hline \multirow[t]{8}{*}{ Ogut $(1996)^{26}$} & \multirow[t]{8}{*}{$\begin{array}{l}\text { Non-randomised } \\
\text { intervention }\end{array}$} & $\begin{array}{l}\text { P } 2.5 \%, \text { C } 0.5 \%, \text { T } 0.5 \% \\
\text { (1 d) }\end{array}$ & 39.4 weeks & 3150 & 10 & $\checkmark$ & \\
\hline & & $\mathrm{P} 2.5 \%, \mathrm{~T} 1 \%(1 \mathrm{~d})$ & 39.4 weeks & 3150 & 10 & $\checkmark$ & \\
\hline & & P 2.5\%, C 1\% (2 d) & 39.4 weeks & 3150 & 10 & $\checkmark$ & \\
\hline & & C $1 \%, T 1 \%(1 \mathrm{~d})$ & 39.4 weeks & 3150 & 10 & $\checkmark$ & \\
\hline & & P $2.5 \%(2 d)$ & 39.4 weeks & 3150 & 10 & $\checkmark$ & \\
\hline & & C $1 \%(2 d)$ & 39.4 weeks & 3150 & 10 & $\checkmark$ & \\
\hline & & T $1 \%(2 d)$ & 39.4 weeks & 3150 & 10 & $\checkmark$ & \\
\hline & & Saline $0.9 \%$ & 39.4 weeks & 3150 & 10 & $\checkmark$ & \\
\hline \multirow{2}{*}{$\begin{array}{l}\text { Phamonvaechavan } \\
(2012)^{23}\end{array}$} & \multirow[t]{2}{*}{ RCT crossover } & P 2.5\%, T $0.75 \%(2 d)$ & 30.5 weeks & 1241.9 & 21 & $\checkmark$ & C \\
\hline & & P 2.5\%, T $0.75 \%(2$ d) & 30.5 weeks & 1241.9 & 21 & $\checkmark$ & C \\
\hline \multirow{2}{*}{$\begin{array}{l}\text { Punyawattanaporn } \\
(2009)^{41}\end{array}$} & \multirow[t]{2}{*}{$\mathrm{RCT}$} & P 1\%, C $0.2 \%(1 \mathrm{~d})$ & $30.49(2.34)$ & 1368 (438.99) & 70 & $\checkmark$ & $\mathrm{R}$ \\
\hline & & P 1\%, C $0.2 \%$ (3 d) & $30.49(2.34)$ & 1368 (438.99) & 70 & $\checkmark$ & $\mathrm{R}$ \\
\hline Rosales $(1981)^{24}$ & $\begin{array}{l}\text { Observational } \\
\text { prospective }\end{array}$ & P 2.5\%, T 0.5\% (3 d) & - & - & 10 & & C \\
\hline Rush $(2004)^{25}$ & $\begin{array}{l}\text { Observational } \\
\text { prospective }\end{array}$ & P 2.5\%, T $0.5 \%$ (3 d) & - & - & 30 & & $\mathrm{R}, \mathrm{C}$ \\
\hline \multirow[t]{3}{*}{ Sindel $(1986)^{19}$} & \multirow[t]{3}{*}{$\mathrm{RCT}$} & P $2.5 \%$, T $1 \%(2$ d) & 28.0 (1.9) weeks & $1022(226)$ & 10 & $\checkmark$ & C \\
\hline & & P 2.5\%, T 0.5\% (2 d) & 28.3 (1.6) weeks & $1115(281)$ & 10 & $\checkmark$ & C \\
\hline & & P 1\%, T 1\% (2 d) & $29.0(2.4)$ weeks & $1110(317)$ & 10 & $\checkmark$ & C \\
\hline \multirow[t]{3}{*}{ Vicente $(2012)^{42}$} & \multirow[t]{3}{*}{$\mathrm{RCT}$} & P 1\%, C $0.2 \%(1 \mathrm{~d})$ & 28.7 (2.6) weeks & - & 5 & $\checkmark$ & \\
\hline & & P 1\%, C $0.2 \%(2$ d) & 28.7 (2.6) weeks & - & 10 & $\checkmark$ & \\
\hline & & P 1\%, C $0.2 \%$ (3 d) & 28.7 (2.6) weeks & - & 15 & $\checkmark$ & \\
\hline \multirow[t]{2}{*}{ Wheatcroft (1993) ${ }^{1}$} & \multirow{2}{*}{$\begin{array}{l}\text { Non-randomised } \\
\text { intervention }\end{array}$} & P $2.5 \%, C$ 0.5\%, (2 d) & 29.8 weeks & 1238 & 26 & $\checkmark$ & \\
\hline & & P $2.5 \%$, C $0.5 \%,(2$ microd $)$ & 29.8 weeks & 1238 & 26 & $\checkmark$ & \\
\hline
\end{tabular}

Efficacy was identified if authorsmeasured pupil dilation or successful retinopathy of prematurity eye examinations. Safety was determined if physiological measurements were taken.

BW, birth weight; C, cardiovascular; C, cyclopentolate;D, dermal; G, gastrointestinal; GA, gestational age; P, phenylephrine; R, respiratory;RCT, randomised controlled trial; T, tropicamide; d, standard drop; microd, microdrop.

3. Clinical significance is difficult to determine due to inability to adjust for confounders and gestational age of infants.

4. No statistically significant changes in blood pressure and heart rate were seen with microdrop administration.

5. Case reports highlight serious adverse effects such as cardiopulmonary arrest and pulseless.

A number of authors have investigated blood pressure and heart rate adverse effects associated with mydriatics (table 4). All results in table 4 are after mydriatic administration, but before ROPEE, which is important as ROPEE is a confounder due to the oculocardio reflex-mediated reduction in heart rate, of approximately $10 \%$, following ROPEE. ${ }^{14}$

Evidence collected from a retrospective audit, with a sample size of 1254 premature infants, showed significant increases in blood pressure after administration of phenylephrine $0.5 \%$ and tropicamide $0.5 \%, 3 \mathrm{~d}$ (mean diastolic blood pressure $8.5 \% \quad(p=0.04)$, mean systolic blood pressure $5.4 \%(\mathrm{p}=0.078)) .{ }^{10}$ This was compared with other cardiovascular related data sets which were from pilot studies, and within these eight different regimens, statistically significant increases in mean blood pressure were seen. The range of these increases in mean blood pressure from baseline ranged from $3.7 \%$ (no SD) to $22.8 \pm 17.4 \%$ (table 4). ${ }^{6} 101516$ Because multiple mydriatic regimens were used in these studies, no causative regimen was identified as having more of an effect on blood pressure.

A reduction in blood pressure was also identified in some studies. Four of these mydriatic regimens in pilot studies found statistically significant reductions in mean blood pressure from baseline, and these ranged from $1 \pm 2.3 \%$ to $17.1 \pm 10.4 \%{ }^{17-19}$ 


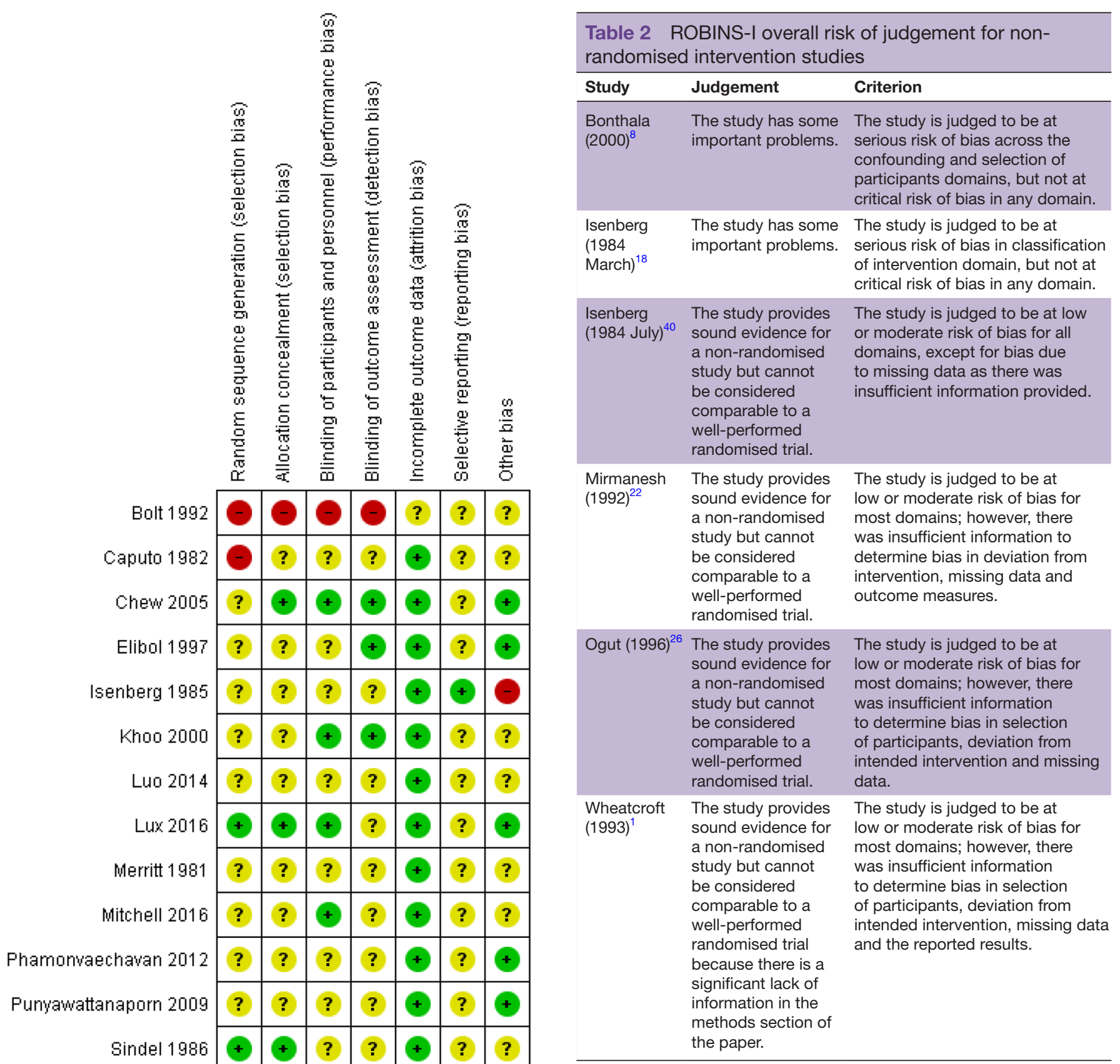

Vicente 2012

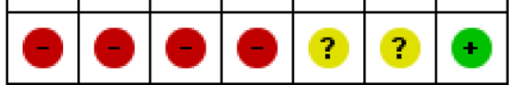

Figure 2 Cochrane tool risk of bias summary for randomised controlled trial (RCT) or quazi-RCT studies. Review authors' judgements about each risk of bias item for each included study. Key: '-' indicates high risk of bias; '?' indicates unclear risk of bias; '+' indicates low risk of bias.

Clinical significance of all of the blood pressure results is difficult to determine without directly comparing the results to the individual gestational age of the infant and adjusting for confounders.

Statistically significant changes in heart rate only occurred when two anticholinergic medicines were used. An increase of the mean heart rate of $3.7 \%$ occurred following cyclopentolate $0.5 \%(1 \mathrm{~d})$ and tropicamide $0.5 \%$ ( $2 \mathrm{~d}$ ) administration, ${ }^{7}$ additionally, an increase in

Table 3 National Heart Lung and Blood Institute overall risk of judgement for observational studies

\begin{tabular}{ll}
\hline Study & Judgement \\
\hline Alshafei $(2017)^{6}$ & Fair \\
\hline Jiang $(2016)^{10}$ & Good \\
Laws $(1996)^{11}$ & Fair \\
Lees $(1981)^{12}$ & Fair \\
Mitchell $(2011)^{46}$ & Good \\
Neffendorf $(2015)^{43}$ & Good \\
Rosales $(1981)^{24}$ & Poor \\
\hline Rush $(2001)^{25}$ & Fair \\
\hline
\end{tabular}




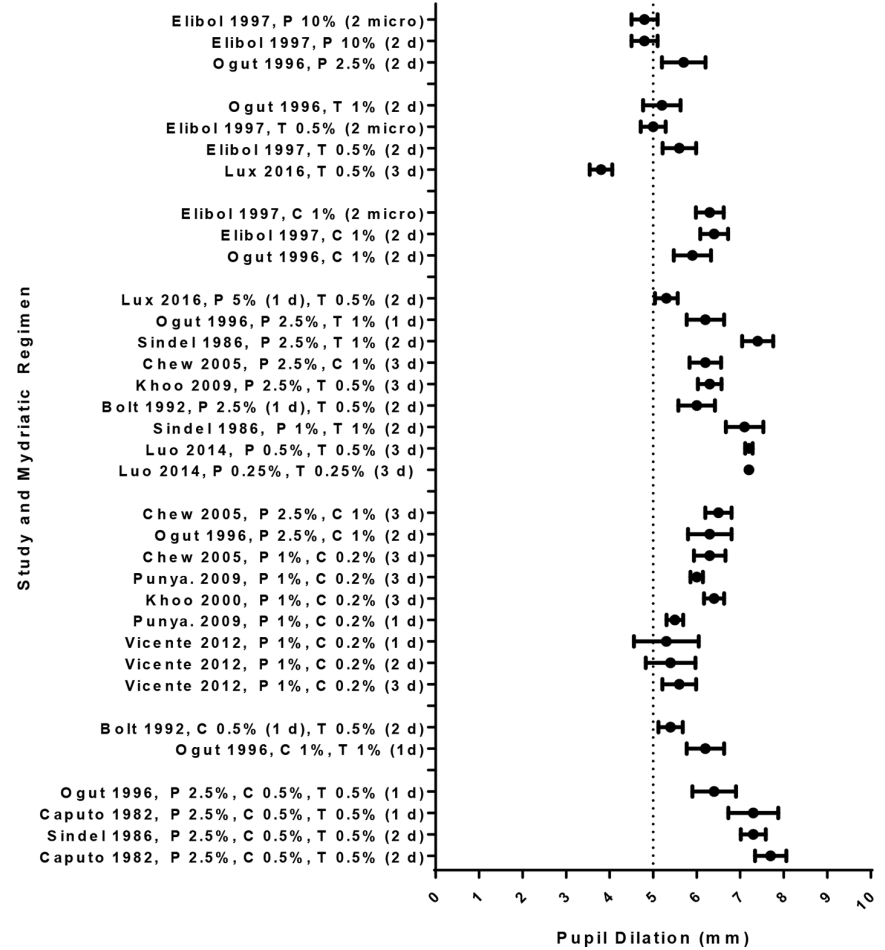

Figure 3 Pupil dilation results (mean and 95\% Cl) for treatment arms in RCTs, including quasi-RCT and crossover RCT. The dotted line is the estimated pupil dilation (5 $\mathrm{mm}$ ) that is required for an ophthalmologist to perform a successful retinopathy of prematurity eye examinations. C, cyclopentolate; d, drop; micro, microdrop; P, phenylephrine; $\mathrm{RCT}$, randomised controlled trial; $\mathrm{T}$, tropicamide.

heart rate of $10 \% \pm 10.6 \%$ occurred following phenylephrine $2.5 \%$, tropicamide $0.5 \%$ and cyclopentolate $0.5 \%$ (2 d) administration. ${ }^{19}$

There were no significant cardiovascular changes recorded in the microdrop groups, which is likely explained by the lower total dose leading to minimal systemic absorption. ${ }^{17}$

Blood pressure and heart rate results from Caputo (1982), ${ }^{9}$ Chew (2005), ${ }^{20}$ Luo (2014), ${ }^{21}$ Mirmanesh (1992), ${ }^{22}$ Pharmonvaechavan (2012) ${ }^{23}$ Rosales (1981), ${ }^{24}$ Rush $(2004)^{25}$ were not able to be included in the analysis because SD or SE and $\mathrm{p}$ values were not included in the publication. Blood pressure results from Rosales (1981) ${ }^{24}$ were not able to be included in the analysis because SD and $p$ values were not included in the publication. Ogut $(1996)^{26}$ results were not able to be included because $\mathrm{p}$ values were not presented. Luo $(2014)^{21}$ commented that no infants had bradycardia, and Lux's $(2016)^{27}$ study design intended to collect data on pulse rate and blood pressure but did not provide results for this.

Very specific cardiovascular monitoring occurred in a study of 42 premature infants, where mean anterior and middle cerebral artery blood flow, heart rate, end diastolic velocity (anterior and middle cerebral artery) and blood pressure was measured. Results from this observational study found no statistically significant results; however, this study may have been underpowered to detect a statistically significant result. ${ }^{6}$

Fourteen cardiovascular adverse events were documented in case reports (table 5). All infants had phenylephrine administered; however, due to the variation in regimens, no themes of likely causative regimens were determined. Tachycardia and bradycardia were the most common reports, and cardiopulmonary arrest and pulseless being the most clinically significant.

\section{Gastrointestinal \\ Key findings}

1. Phenylephrine can cause vasoconstriction of the blood supply to the gut, and anticholinergic effect on the gut reduces peristalsis; hence, both mechanisms of actions affect the gastrointestinal system.

2. Feed intolerance, abdominal distention, ileus and NEC are gastrointestinal adverse effects associated with mydriatic use.

3. All cases of gastrointestinal adverse effects had phenylephrine administered, and the majority had cyclopentolate. Only one case involved the use of tropicamide.

4. Three infants have died with complications associated with NEC, possibly linked with mydriatic use.

5. All safety and case reports had regimens where standard drops were used.

Reduced gastric volume, increased abdominal girth, increased feed intolerance and increased incidence of NEC were some of the gastrointestinal adverse effects investigated by authors (table 6). Additionally, Jiang (2016) ${ }^{10}$ found that approximately $10 \%$ of infants developed NEC following phenylephrine $0.5 \%$ and tropicamide $0.5 \%$ (3d) administration $(p<0.01)$, and new cases of upper digestive tract haemorrhage also increased $(\mathrm{n}=139, \mathrm{p}<0.00) .{ }^{10}$

Antral and duodenal motor activity and gastric emptying, pre and post phenylephrine $2.5 \%$ and cyclopentolate $0.5 \%$ (2 d) administration was measured in 11 premature infants. ${ }^{8}$ Duodenal motor contractions decreased nearly fourfold after mydriatic instillation and gastric emptying was significantly delayed (table 5 ). ${ }^{8}$

Results from an intervention study $(n=10)$, demonstrated a reduction in gastric acid secretion of approximately $58 \%$ following cyclopentolate $0.5 \%$ administration $(\mathrm{p}<0.01)$ (table 6). ${ }^{15}$ This is compared with a RCT $(\mathrm{n}=25)$, where gastric residuals increased following phenylephrine $1 \%$ and cyclopentolate $0.2 \%(3 \mathrm{~d})$ administration $(\mathrm{p}=0.01)$ (table 6). ${ }^{28}$

No statistically significant results from a RCT $(n=39)$ for abdominal girth or feed intolerance occurred following the administration of phenylephrine $2.5 \%$ and cyclopentolate $1 \%$ (3 d) or phenylephrine $2.5 \%$ and tropicamide $1 \%$ (3 d) or phenylephrine $1 \%$ and cyclopentolate $0.2 \%(3 \mathrm{~d}) .{ }^{20}$

Abdominal distention $(n=7)$, NEC $(n=4)$, transient ileus $(n=3)$ and feed intolerance $(n=3)$ are the most common adverse effects documented in case reports (table 7 ). Three infants died following complications associated with NEC. ${ }^{29-31}$ 
Open access

Table 4 Blood pressure and heart rate adverse effects for studies with $p$ values, percentage change from baseline

\begin{tabular}{|c|c|c|c|c|c|}
\hline Paper & Regimen & $\mathrm{mBP}(\mathrm{mm} \mathrm{Hg}) \pm \mathrm{SD}$ & $\mathrm{mDBP}(\mathrm{mm} \mathrm{Hg}) \pm \mathrm{SD}$ & $\mathrm{mSBP}(\mathrm{mm} \mathrm{Hg}) \pm \mathrm{SD}$ & mHR (beats/min) $\pm S D$ \\
\hline Bonthala $(2000)^{8}$ & P 1\%, C $0.2 \%$ (2 d) & $\downarrow 11.5 \pm 5.8 \%{ }^{*}$ & - & - & $\uparrow 0.6 \pm 1.2 \%{ }^{*}$ \\
\hline \multirow[t]{2}{*}{ Bolt $(1992)^{7}$} & P 2.5\% (1d), T $0.5 \%$ (2 d) & $\downarrow 4 \%$ & - & - & $\downarrow 1 \%$ \\
\hline & C $0.5 \%$ (1d), T $0.5 \%$ (2 d) & $\uparrow 5.1 \% *$ & - & - & $\uparrow 3.7 \% \dagger$ \\
\hline \multirow[t]{6}{*}{ Elibol $(1997)^{17}$} & C $1 \%(2 d)$ & $\uparrow 16.2 \% \pm 2.2 \%{ }^{*} \dagger$ & - & - & $\ddagger$ \\
\hline & C $1 \%$ (2 microd) & $\downarrow 1 \% \pm 2.3 \%{ }^{*} \dagger$ & - & - & $\ddagger$ \\
\hline & P 10\% (2 d) & $\uparrow 8 \% \pm 0.2 \%{ }^{*} \dagger$ & - & - & $\ddagger$ \\
\hline & P 10\% (2 microd) & $0 \pm 1.9 \% *$ & - & - & $\ddagger$ \\
\hline & T $0.5 \%(2 d)$ & $\uparrow 17.5 \% \pm 0.2 \%{ }^{*} \dagger$ & - & - & $\ddagger$ \\
\hline & T 0.5\% (2 microd) & $\downarrow 1.4 \pm 0.3 \%{ }^{*}$ & - & - & $\ddagger$ \\
\hline \multirow[t]{3}{*}{ Isenberg (1984 March) ${ }^{40}$} & C $0.5 \%(2 d)$ & $\downarrow 10 \% \pm 12 \% \dagger$ & - & - & $\uparrow 7.5 \% \pm 18 \%$ \\
\hline & C $0.5 \%$, T $0.5 \%$ (2 d) & $\downarrow 10 \% \pm 12 \% \dagger$ & - & - & $\uparrow 7.5 \% \pm 18 \%$ \\
\hline & P 1\%, C 0.2\% (2 d) & $\downarrow 5.8 \% \pm 14 \% \dagger$ & - & - & $\downarrow 1.7 \% \pm 11 \%$ \\
\hline \multirow[t]{3}{*}{ Isenberg (1984 July) $)^{18}$} & C $0.5 \%$ & $\downarrow 13.2 \%$ & & & $\downarrow 3.3 \%$ \\
\hline & P $1 \%$, C $0.2 \%$ & $\downarrow 3.8 \%$ & & & $\downarrow 4.3 \%$ \\
\hline & P 2.5\%, T 0.5\% & $\uparrow 19.6 \%$ & & & $\downarrow 5.2 \%$ \\
\hline Jiang $(2016)^{10}$ & P $0.5 \%, T 0.5 \%$ (3 d) & - & $\uparrow 8.5^{\star} \dagger$ & $\uparrow 5.4 \% * \S$ & - \\
\hline \multirow[t]{2}{*}{ Khoo $(2000)^{16}$} & P 1\%, C $0.2 \%$ (3 d) & $\uparrow 3.7 \%$ & $-1 \%$ & $\uparrow 0.8 \%$ & $\downarrow 4.5 \%$ \\
\hline & P 2.5\%, T 0.5\% (3 d) & $\downarrow 0.4 \%$ & $\downarrow 0.3 \%$ & $\downarrow 1.2 \%$ & $\downarrow 6.6 \%$ \\
\hline Laws $(1996)^{11}$ & P 2.5\% (2d), C $0.5 \%$ (4 d) & - & $\uparrow 4.8 \%^{*}$ & $\uparrow 5 \% *$ & $\downarrow 1.3 \%^{*}$ \\
\hline Lees $(1981)^{12}$ & P $2.5 \%, T 0.5 \%$ (1 d) & $\uparrow 19.4 \% \dagger$ & $\uparrow 19.1 \% \dagger$ & $\uparrow 17.1 \% \dagger$ & $0 \%$ \\
\hline \multirow[t]{2}{*}{ Merritt $(1981)^{45}$} & P 2.5\%, T 1\% (3 d) & - & - & $0 \%$ & - \\
\hline & $\begin{array}{l}\text { P } 2.5 \%, \text { T } 0.5 \%, \\
\text { C } 0.5 \% \text { (3 d) }\end{array}$ & - & - & $0 \%$ & - \\
\hline $\begin{array}{l}\text { Phamonvaechavan } \\
(2012)^{23}\end{array}$ & P $2.5 \%, T 0.75 \%$ (2 d) & - & $0 \%$ & $0 \%$ & $\downarrow 3.2 \%$ \\
\hline \multirow[t]{3}{*}{ Sindel $(1986)^{19}$} & P 2.5\%, T 1\% (2 d) & $\downarrow 17.1 \% \pm 10.4 \% \dagger$ & - & - & - \\
\hline & $\begin{array}{l}\text { P } 2.5 \%, \text { T } 0.5 \%, \\
\text { C } 0.5 \% \text { (2 d) }\end{array}$ & $\uparrow 22.8 \% \pm 17.4 \% \dagger$ & - & - & $\uparrow 10.0 \% \pm 10.6 \% \dagger$ \\
\hline & P 1\%, T 1\% (2 d) & $\uparrow 7.7 \% \pm 9.3 \% \dagger$ & - & - & - \\
\hline
\end{tabular}

*Data approximated from graph or was converted to percentage.

†P $<0.05$ to 0.01 .

$\ddagger$ Data not presented.

$\S \mathrm{P}=0.08$.

$\mathrm{C}$, cyclopentolate; P, phenylephrine; T, tropicamide; d, standard drop;mDBP, mean diastolic blood pressure; mHR, mean heart rate; mSDP, mean systolic blood pressure; microd, microdrop.

\section{Respiratory}

Key findings

1. Unwanted respiratory effects could occur via alpha-agonist mediated activity. Phenylephrine has a stimulatory effect on increasing airway smooth muscle tension, which leads to bronchoconstriction, reduced oxygen saturations and apnoea.

2. Infants with bronchopulmonary dysplasia (BPD) are more likely to experience respiratory adverse effects compared with those without BPD.

\begin{tabular}{|c|c|c|c|c|}
\hline Paper & Regimen & Tachycardia & Bradycardia & Other \\
\hline Ahmad (2016) ${ }^{47}$ & P 1\%, C 0.2\%, (unknown d) & 2 & 2 & Premature atrial contractions (2) \\
\hline Ozgun $(2014)^{29}$ & P $1.25 \%, C 0.5 \%(2 d)$ & 1 & & \\
\hline Socarras $(2017)^{48}$ & P 2.5\%, T(unknown concentration) (2 d) & 1 & & Arterial hypertension (1) \\
\hline Ahmad $(2016)^{47}$ & P 1\%, C0.2\% (3 d) & & & Cardiopulmonary arrest (1) \\
\hline Wood $(2009)^{49}$ & P $1 \%, C$ 0.2\% (1-2 d) & & 2 & Pulseless (1) \\
\hline $\operatorname{Siu}(2011)^{30}$ & P 2.5\%, C 1\% (2 d) & & 1 & \\
\hline
\end{tabular}

C, cyclopentolate;P, phenylephrine; T, tropicamide; d, standard drop. 
Table 6 Publications providing data on gastric volume, abdominal girth and feed intolerance

\begin{tabular}{|c|c|c|c|c|}
\hline Paper & Regimen & $\begin{array}{l}\text { Mean gastric volume } \pm \text { SD ( } \mu \mathrm{mol} / \\
\mathrm{kg} / \mathrm{hour} \text { ) }\end{array}$ & $\begin{array}{l}\text { Abdominal girth } 24 \text { hours post } \\
\text { (cm) }\end{array}$ & $\begin{array}{l}\text { Feed } \\
\text { intolerance }\end{array}$ \\
\hline \multirow[t]{3}{*}{ Chew $(2005)^{20}$} & P 2.5\%, C 1\% (3 d) & - & $25.2 \pm 1.6$ & $50 \%$ \\
\hline & P 2.5\%, T 1\% (3 d) & - & $27.1 \pm 2.0$ & $25 \%$ \\
\hline & P 1\%, C $0.2 \%$ (3 d) & - & $27.1 \pm 2.9$ & $25 \%$ \\
\hline \multirow[t]{2}{*}{ Isenberg $(1985)^{15}$} & C $0.25 \%(2 \mathrm{~d})$ & $10 \pm 7$ & - & - \\
\hline & C $0.5 \%(2 d)$ & Approx. $2.5 \pm 1^{*}$ & - & - \\
\hline Mitchell $(2016)^{28}$ & P 1\%, C $0.2 \%$ (3 d) & $\begin{array}{l}\text { Increased gastric residual } \\
\text { (statistically significant)† }\end{array}$ & - & - \\
\hline
\end{tabular}

${ }^{*} \mathrm{P}<0.01$.

†Data not presented.

C, cyclopentolate;P, phenylephrine; T, tropicamide; d, standard drop.

3. Infants on oxygen support are more likely to have higher serum concentrations of cyclopentolate than those not on oxygen support.

4. The most common adverse effects identified in case reports are apnoea and desaturation.

5. All safety and case reports had regimens where standard drops were used.

\begin{tabular}{|c|c|c|c|c|}
\hline Paper & Regimen & $\begin{array}{l}\text { NEC } \\
\text { and } \\
\text { death }\end{array}$ & NEC & Other \\
\hline CARM $(2014)^{31}$ & $\begin{array}{l}\text { P } 2.5 \%, C \text { C } 0.5 \% \\
\text { (3 d) }\end{array}$ & 1 & & \\
\hline Ozgun $(2014)^{29}$ & $\begin{array}{l}P 1.25 \%, C 0.5 \% \\
(2 \text { d) }\end{array}$ & 1 & & $\begin{array}{l}\text { Abdominal } \\
\text { distention (1) }\end{array}$ \\
\hline Siu $(2011)^{30}$ & P $2.5 \%, C 1 \%(2$ d) & 1 & 1 & $\begin{array}{l}\text { Abdominal } \\
\text { distension (1) } \\
\text { Milk } \\
\text { intolerance (1) } \\
\text { Pneumatosis } \\
\text { intestinalis (1) }\end{array}$ \\
\hline CARM $(2002)^{35}$ & P 1\%, C 0.5\% (1 d) & & & $\begin{array}{l}\text { Abdominal } \\
\text { distention (1) }\end{array}$ \\
\hline $\begin{array}{l}\text { Degrimencioglu } \\
(2014)^{50}\end{array}$ & $\begin{array}{l}\text { P 2.5\%, T } 0.5 \% \\
\text { (3 d) }\end{array}$ & & & $\begin{array}{l}\text { Feed } \\
\text { intolerance (1) } \\
\text { Abdominal } \\
\text { distension (1) } \\
\text { Transient } \\
\text { ileus (1) }\end{array}$ \\
\hline Socarras $(2017)^{48}$ & $\begin{array}{l}\text { P 2.5\%, } \\
\text { T(unknown\%) (2 d) }\end{array}$ & & & $\begin{array}{l}\text { Feed } \\
\text { intolerance (1) } \\
\text { Abdominal } \\
\text { distension (1) }\end{array}$ \\
\hline Sarici $(2001)^{51}$ & $\begin{array}{l}P 2.5 \%, C \text { 0.5\% } \\
\text { (2 d) }\end{array}$ & & & $\begin{array}{l}\text { Vomiting (1) } \\
\text { Abdominal } \\
\text { distention (1) } \\
\text { Gastric } \\
\text { dilation (1) }\end{array}$ \\
\hline $\operatorname{Lim}(2003)^{52}$ & P 1\%, C 0.2\% (3 d) & & & $\begin{array}{l}\text { Abdominal } \\
\text { distention (1) } \\
\text { Transient } \\
\text { ileus (1) }\end{array}$ \\
\hline $\operatorname{Lim}(2003)^{52}$ & P 1\%, C $0.2 \%$ (3 d) & & & $\begin{array}{l}\text { Transient } \\
\text { ileus (1) }\end{array}$ \\
\hline
\end{tabular}

C, cyclopentolate; CARM, Centre for Adverse Reactions Monitoring; NEC, necrotising enterocolitis; $P$, phenylephrine; $\mathrm{T}$, tropicamide; $\mathrm{d}$, standard drop.
Oxygen saturation and apnoea effects were investigated in just a few studies (table 8 ). Results from a pilot observational study $(n=42)$ demonstrated no change in oxygen saturations following the use of phenylephrine $2.5 \%$ and tropicamide $0.5 \%$ (3 d) ( $>0.05)$ (table 8). This compares audit data from 1183 infants, where significant increases in apnoea were seen following significantly lower doses of phenylephrine $0.5 \%$ and equal dose of tropicamide $0.5 \%(3 \mathrm{~d})(\mathrm{p}<0.05)($ table 6$) .{ }^{10}$

Following phenylephrine 2.5\% (3 d) administration, infants with BPD are more likely to experience a reduction in mean pulmonary compliance $(\mathrm{p}<0.03)$, increased mean total resistance $(\mathrm{p}<0.01)$ and reduced expiratory airflow $(\mathrm{p}<0.03)$, compared with infants without BPD. ${ }^{22}$

Cyclopentolate serum concentrations were measured following cyclopentolate $0.2 \%$ (3 d) administration. $^{28}$ Results show that infants who were receiving oxygen therapy, had significantly higher cyclopentolate concentrations, than infants who were not receiving oxygen therapy $(\mathrm{p}=0.01)$. The infants with higher cyclopentolate

Table 8 Publications providing data on respiratory adverse effects

\begin{tabular}{|c|c|c|}
\hline Paper & Regimen & Adverse effect \\
\hline Alshafei $(2017)^{6}$ & $\begin{array}{l}P 2.5 \%, T \\
0.5 \%(3 \mathrm{~d})\end{array}$ & $\begin{array}{l}\text { No adverse effect on oxygen } \\
\text { saturation, not statistically } \\
\text { significant }\end{array}$ \\
\hline Jiang $(2016)^{10}$ & $\begin{array}{l}\text { P } 0.5 \%, T \\
0.5 \%(3 \mathrm{~d})\end{array}$ & $\begin{array}{l}\text { Apnoea: statistically } \\
\text { significant increase }(p<0.05) \text {. }\end{array}$ \\
\hline Mitchell $(2016)^{28}$ & $\begin{array}{l}P 1 \%, C 0.2 \% \\
(3 \mathrm{~d})\end{array}$ & $\begin{array}{l}\text { Desaturations more likely } \\
\text { in oxygen therapy group } \\
(p=0.01)\end{array}$ \\
\hline Mirmanesh (1992) & P 2.5\% (3 d) & $\begin{array}{l}\text { Infants with BPD; } \\
\begin{array}{l}\text { Reduction in mean } \\
\text { pulmonary compliance } \\
(p<0.03) \\
\text { Increased mean total } \\
\text { resistance }(\mathrm{p}<0.01) \\
\text { Reduced expiratory airflow } \\
(p<0.03)\end{array}\end{array}$ \\
\hline $\begin{array}{l}\text { Punyawattanporn } \\
(2009)^{41}\end{array}$ & Not identified & Apnoea: one infant. \\
\hline
\end{tabular}

C, cyclopentolate;P, phenylephrine; T, tropicamide; d, standard drop. 


\begin{tabular}{|c|c|c|c|c|}
\hline Paper & Regimen & Apnoea & Desaturation & Other \\
\hline Sarici $(2001)^{51}$ & P $2.5 \%, C$ 0.5\% (2 d) & 1 & & \\
\hline Degirmencioglu (2014) & P 2.5\%, T 0.5\% (3 d) & 1 & & \\
\hline Wood $(2009)^{49}$ & P 1\%, C 0.2\% (1-2 d) & 1 & & Shallow breathing (1) \\
\hline Wood $(2009)^{49}$ & P 1\%, C $0.2 \%(1-2 d)$ & 1 & & \\
\hline CARM $(2002)^{35}$ & P 1\%, C 0.5\% (1 d) & 1 & 1 & \\
\hline Siu $(2011)^{30}$ & P $2.5 \%, C 1 \%(2 d)$ & & 1 & \\
\hline $\operatorname{Kim}(2015)^{53}$ & P $2.5 \%$, T $0.5 \%$ (2 d) & & 1 & Mild wheezing (1) \\
\hline $\operatorname{Kim}(2015)^{53}$ & P 2.5\%, T 0.5\% (2 d) & & & $\begin{array}{l}\text { Bronchospasm (1) } \\
\text { Mild wheezing (1) }\end{array}$ \\
\hline Ozgun $(2014)^{29}$ & P $1.25 \%, C 0.5 \%$ (2 d) & & & Tachyapnoea (1) \\
\hline
\end{tabular}

C, cyclopentolate;P, phenylephrine; $\mathrm{T}$, tropicamide; $\mathrm{d}$, standard drops.

concentrations and who were receiving oxygen therapy also were more likely to have desaturations $(p=0.01){ }^{28}$

Lux's $(2016)^{27}$ study design intended to collect data on oxygen saturation and respiratory frequency but did not provide results for this. ${ }^{27}$

Thirteen respiratory associated events are documented with the most common are apnoea $(n=5)$, desaturations $(n=3)$ and other respiratory events $(n=5)($ table 9$)$.

\section{Central nervous system}

No safety data on CNS adverse effects has been published. The only CNS data available are case reports and involve seizures $(n=5)$ and anticholinergic syndrome $(n=1){ }^{31-34}$ All cases involved the use of cyclopentolate $0.5 \%$. The mechanism for seizure activity following alpha agonist and anticholinergic agents is unknown.

\section{Dermal}

Three infants experienced periorbital pallor following phenylephrine $2.5 \%$ and tropicamide $0.5 \%\left(\begin{array}{ll}1 & \mathrm{~d}\end{array}\right){ }^{12}$ Conversely, skin flushing was observed following cyclopentolate $1 \%(2 \mathrm{~d})$ and phenylephrine $10 \%$ (2 d) administration. $^{17}$

A mechanism for skin flushing is unknown, and skin flushing seems very unusual considering the mechanism of action of phenylephrine. If phenylephrine eye drop excess is not wiped away from the eye area, this allows for phenylephrine to be absorbed dermally. Subsequent vasoconstriction occurs which results in periorbital pallor.

Ten dermal events have been reported, and these include eyelid swelling $(n=4)$ and periorbital pallor $(n=6) .{ }^{35-38}$

\section{Other}

There were a few other case reports identified which did not fit into the above themes, and the cases involved cutis marmorata, leukopaenia, neutropaenia and renal failure. ${ }^{29} 39$

\section{DISCUSSION}

This review has identified that low dose mydriatics have comparable efficacy to larger doses; therefore, irrespective of mydriatic regimen, sufficient pupil dilation occurs at low doses.

Of all the regimens reviewed for efficacy, phenylephrine $1 \%$ and cyclopentolate $0.2 \%(1-2 \mathrm{~d})$ is the lowest combination dose that provides sufficient pupil dilation. ${ }^{40-42}$ However, phenylephrine $2.5 \%$ and cyclopentolate $0.5 \%(2-3 \mathrm{~d})$ is the recommended regimen in the UK guideline, ${ }^{3}$ although there is no known publication evaluating the efficacy of the three-drop regimen. This dose seems unnecessarily high considering the current body of knowledge about efficacy. Regarding safety, results from a retrospective audit of 138 premature infants who underwent ROPEE's using the UK guideline regimen, highlighted six cases of harm ranging from apnoea, desaturations and $\mathrm{NEC}^{43}$ there is an unpublished case of death associated with NEC $^{31}$ and one reported case of a seizure. ${ }^{33}$

Although the extent of mydriatic harm has not been fully investigated, a few studies signal the potential for cardiovascular, gastrointestinal and respiratory adverse effects. It is very likely that our most vulnerable premature infants with pre-existing medical conditions are the ones who are more likely to experience adverse effects associated with mydriatics and are less likely to be able to compensate for any medicine related harms. Medical practice is evolving in both the developed and low and middle-income countries, and as the ROPEE standard of care is being extended, it is likely that there will be an increase in adverse effects post-ROPEE in centres who are using high-dose mydriatic regimens.

Simple concepts that need further investigations are giving low concentrations as a single microdrop. This method would reduce mydriatic exposure to the systemic circulation. ${ }^{142}$

Infants with dark irides may also need special dosing consideration, as it is hypothesised that higher doses may need to be administered in people with dark pigmented iris. ${ }^{16} 2044$ This implication for dosing requirements could also have potential increased risks associated with side effects and subsequent harm. Currently, international guidelines do not offer dose adjustment recommendations based on iris colour. 
Further research is required to ascertain this potential association.

And lastly, readily available commercial products are prepared at higher concentrations than what is needed, and current packaging is suitable for adult dose administration and not neonatal dosing. Further research on appropriate formulations and packaging for commercial products needs to occur.

\section{Limitations}

Limitations of this systematic review are (1) the non-randomised trials included in the review were not designed as comparative studies, (2) case reports were assessed as reported and no independent assessment was carried out for causality, (3) there was a significant amount of unclear bias in the publications and (4) papers not available in English were not included in this review.

An assumption was made that if microdrop administration was not specified and that standard drops were administered.

Confounders, such as pre-existing medical conditions and pain associated with ROPEE, were often not mentioned in the publications included in the analysis.

Number of case reports are likely to be much higher. It is estimated that approximately $10 \%$ of adverse drug reactions are ever reported, and very few of these make it to publication.

\section{CONCLUSIONS}

Current international dosing guidance seems unnecessarily high, especially when the reviewed literature suggest that lower doses are effective. All studies investigating efficacy were underpowered due to limited sample size; however, the lowest effective regimen appears to be phenylephrine $1 \%$ and cyclopentolate $0.2 \%(1 \mathrm{~d}) .{ }^{42}$ Microdrop administration of this and other regimens would further increase the safety profile, . howeverfurther investigation and validation needs to occur.

In conclusion, low doses of mydriatics appear to be effective and are likely to be associated with a safer adverse effect profile.

Acknowledgements The authors would like to thank Janelle Ashton (CARM) and Dr Carolyn Coulter.

Contributors RB suggested the topic for the review. LK carried out the review, planned the structure of the article and conducted the systematic review and wrote the article. RB, DR and NM provided comments, supervision and support throughout.

Funding The authors have not declared a specific grant for this research from any funding agency in the public, commercial or not-for-profit sectors.

Disclaimer The views expressed in the submitted article are our own and not an official position of the institution.

Competing interests None declared.

Patient consent for publication Not required.

Provenance and peer review Not commissioned; externally peer reviewed.

Data availability statement Data are available in a public, open access repository.
Open access This is an open access article distributed in accordance with the Creative Commons Attribution Non Commercial (CC BY-NC 4.0) license, which permits others to distribute, remix, adapt, build upon this work non-commercially, and license their derivative works on different terms, provided the original work is properly cited, appropriate credit is given, any changes made indicated, and the use is non-commercial. See: http://creativecommons.org/licenses/by-nc/4.0/.

\section{REFERENCES}

1. Wheatcroft $S$, Sharma A, McAllister J. Reduction in mydriatic drop size in premature infants. Br J Ophthalmol 1993;77:364-5.

2. Shell JW. Pharmacokinetics of topically applied ophthalmic drugs. Surv Ophthalmol 1982;26:207-18.

3. Royal College of Ophthalmologists, Royal College of Paediatricsand Child Health. Guideline for the screening and treatment of retinopathy of prematurity, 2008.

4. Fierson WM, American Academy of Pediatrics Section on Ophthalmology, American Academy of Ophthalmology, et al. Screening Examination of premature infants for retinopathy of prematurity. Pediatrics 2013;131:189-95.

5. National Heart $L$ and Blood Institute. Study quality assessment tools [Internet]. Bethesda, MD: National Institute of Health, 2019. Available: https://www.nhlbi.nih.gov/health-topics/study-qualityassessment-tools [Accessed 27 Mar 2019].

6. Alshafei A, Ahmed M, Farouk S, et al. Impact of mydriatic eye drops on neonatal cerebral blood flow. Journal of Pediatric and Neonatal Individualized Medicine 2017;7.

7. Bolt B, Benz B, Koerner F, et al. A mydriatic eye-drop combination without systemic effects for premature infants: a prospective doubleblind study. J Pediatr Ophthalmol Strabismus 1992;29:157-62.

8. Bonthala S, Sparks JW, Musgrove KH, et al. Mydriatics slow gastric emptying in preterm infants. J Pediatr 2000;137:327-30.

9. Caputo AR, Schnitzer RE, Lindquist TD, et al. Dilation in neonates: a protocol. Pediatrics 1982;69:77-80.

10. Jiang J-B, Zhang Z-W, Zhang J-W, et al. Systemic changes and adverse effects induced by retinopathy of prematurity screening. Int J Ophthalmol 2016;9:1148-55.

11. Laws DE, Morton C, Weindling M, et al. Systemic effects of screening for retinopathy of prematurity. $\mathrm{Br} \mathrm{J} O p h t h a l m o l$ 1996;80:425-8.

12. Lees BJ, Cabal LA. Increased blood pressure following pupillary dilation with $2.5 \%$ phenylephrine hydrochloride in preterm infants. Pediatrics 1981;68:231-4.

13. Children NZFf. New Zealand formulary for children dunedin, New Zealand, 2018. Available: https://nzfchildren.org.nz/nzf_1 accessed 28.09.182018 [Accessed Sep 01 2018].

14. Young TE. Topical mydriatics: the adverse effects of screening examinations for retinopathy of prematurity. Pharmacology Review 2003;4:e163-6

15. Isenberg SJ, Abrams C, Hyman PE. Effects of cyclopentolate eyedrops on gastric secretory function in pre-term infants. Ophthalmology 1985;92:698-700.

16. Khoo BK, Koh A, Cheong P, et al. Combination cyclopentolate and phenylephrine for mydriasis in premature infants with heavily pigmented irides. J Pediatr Ophthalmol Strabismus 2000;37:15-20.

17. Elibol O, Alçelik T, Yüksel N, et al. The influence of drop size of cyclopentolate, phenylephrine and tropicamide on pupil dilatation and systemic side effects in infants. Acta Ophthalmol Scand 1997;75:178-80.

18. Isenberg S, Everett S. Cardiovascular effects of mydriatics in lowbirth-weight infants. J Pediatr 1984;105:111-2.

19. Sindel BD, Baker MD, Maisels MJ, et al. A comparison of the pupillary and cardiovascular effects of various mydriatic agents in preterm infants. J Pediatr Ophthalmol Strabismus 1986;23:273-6.

20. Chew C, Rahman RA, Shafie SM, et al. Comparison of mydriatic regimens used in screening for retinopathy of prematurity in preterm infants with dark irides. J Pediatr Ophthalmol Strabismus 2005;42:166-73.

21. Luo S, Wan Z, Yin X, et al. Comparison of the mydriatic effects of mydrin-P and compound tropicamide in the screening of retinopathy of prematurity. Eye Sci 2014;29:219-22.

22. Mirmanesh SJ, Abbasi S, Bhutani VK. Alpha-adrenergic bronchoprovocation in neonates with bronchopulmonary dysplasia. $J$ Pediatr 1992;121:622-5.

23. Phamonvaechavan $\mathrm{P}$, Chutasmit K, Damrongrak $\mathrm{P}$, et al. Comparison of the effectiveness of mydriasis by two instillation methods of combined $0.75 \%$ tropicamide and $2.5 \%$ phenylephrine eye drop in preterm infants. J Med Assoc Thai 2012;95 Suppl 4:S1-7.

24. Rosales T, Isenberg S, Leake R, et al. Systemic effects of mydriatics in low weight infants. $J$ Pediatr Ophthalmol Strabismus 1981;18:42-4. 
25. Rush R, Rush S, Nicolau J, et al. Systemic manifestations in response to mydriasis and physical examination during screening for retinopathy of prematurity. Retina 2004;24:242-5.

26. Oğüt MS, Bozkurt N, Özek E, et al. Effects and side effects of mydriatic eyedrops in neonates. Eur J Ophthalmol 1996;6:192-6.

27. Lux A-L, Degoumois A, Barjol A, et al. Combination of $5 \%$ phenylephrine and $0.5 \%$ tropicamide eyedrops for pupil dilation in neonates is twice as effective as $0.5 \%$ tropicamide eyedrops alone. Acta Ophthalmol 2017;95.

28. Mitchell A, Hall RW, Erickson SW, et al. Systemic absorption of cyclopentolate and adverse events after retinopathy of prematurity exams. Curr Eye Res 2016:1-7.

29. Ozgun U, Demet T, Ozge KA, et al. Fatal necrotising enterocolitis due to mydriatic eye drops. J Coll Physicians Surg Pak 2014;24 Suppl 2:S147-9.

30. Siu LY, Chan WH, SK A, et al. Necrotising enterocolitis following the use of mydriatics: a case report of two triplets. Hong Kong Journal of Paediatrics 2011;16:47-50.

31. Centre for Adverse Reactions Monitoring (CARM). Phenylephrine $2.5 \%$ and cyclopentolate $0.5 \%$ adverse effects. Case Report 111811. Dunedin Centre for Adverse Reactions Monitoring, University of Otago; 2014.

32. Centre for Adverse Reactions Monitoring (CARM). Cyclopentolate $0.5 \%$ adverse effects. Case Report 94819. Dunedin Centre for Adverse Reactions Monitoring, University of Otago; 2011.

33. Büyükcam A, Celik HT, Korkmaz A, et al. Myoclonic seizure due to cyclopentolate eye drop in a preterm infant. Turk J Pediatr 2012;54:419-20.

34. Bedirli N, Akgün F, Hondur A, et al. Suspected central anticholinergic syndrome related to cycloplegic eye drop in a premature baby. Balkan Med J 2012;29:326-7.

35. Centre for Adverse Reactions Monitoring (CARM). Phenylephrine $1 \%$ and cyclopentolate $0.5 \%$ adverse effects. Case Report 54093. Dunedin Centre for Adverse Reactions Monitoring, University of Otago; 2002

36. Alpay A, Ermis B, Ugurbas SC, et al. The local vasoconstriction of infant's skin following instillation of mydriatic eye drops. Eur J Clin Pharmacol 2010;66:1161-4.

37. Sesham R, Puvanachandra N, Muthukumar P. Phenylephrine eye drops: a rare complication in preterm neonate. Arch Dis Child Fetal Neonatal Ed 2014;99

38. Alvarado Socarras JL, Rodríguez SC. [Periorbital pallor post application of mydriatic in infants with hydrocephalus associated to systemic effects]. Rev Chil Pediatr 2017;88:280-4

39. Shinomiya K, Kajima M, Tajika H, et al. Renal failure caused by eyedrops containing phenylephrine in a case of retinopathy of prematurity. J Med Invest 2003;50:203-6.
40. Isenberg S, Everett S, Parelhoff E. A comparison of mydriatic eyedrops in low-weight infants. Ophthalmology 1984;91:278-9.

41. Punyawattanaporn A, Tengtrisorn S, Sangsupawanich P. Pupil dilatation after single and triple doses of mydriatic agent in preterm infants. J Med Assoc Thai 2009;92:1458-62.

42. Vicente GV, Bahri M, Palafoutas JJ, et al. A randomized controlled trial to determine the lowest effective dose for adequate mydriasis in premature infants. J Aapos 2012;16:365-9.

43. Neffendorf JE, Mota PM, Xue K, et al. Efficacy and safety of phenylephrine $2.5 \%$ with cyclopentolate $0.5 \%$ for retinopathy of prematurity screening in 1246 eye examinations. Eur J Ophthalmol 2015;25:249-53.

44. Anderson HA, Bertrand KC, Manny RE, et al. Comparison of two drug combinations for dilating dark irides. Optom Vis $\mathrm{Sci}$ 2010;87:120-4.

45. Merritt JC, Kraybill EN. Effect of mydriatics on blood pressure in premature infants. J Pediatr Ophthalmol Strabismus 1981;18:42-6.

46. Mitchell AJ, Green A, Jeffs DA, et al. Physiologic effects of retinopathy of prematurity screening examinations. Adv Neonatal Care 2011;11:291-7.

47. Ahmad A, Mondal T, Klein B. Atrial arrhythmia after newborn eye exam, to caffeine or not to caffeine?. J Neonatal Perinatal Med 2016:9:427-31.

48. Socarras JLA, Rodriguez SC. Periorbital pallor and systemic effects post application of mydriatics in infants with hydrocephalus. Revista Chilena De Pediatria-Chile 2017;88:280-4.

49. Wood MG, Kaufman LM. Apnea and bradycardia in two premature infants during routine outpatient retinopathy of prematurity screening. J Aapos 2009;13:501-3.

50. Degirmencioglu H, Oncel MY, Calisici E, et al. Transient ileus associated with the use of mydriatics after screening for retinopathy of prematurity in a very low birth weight infant. J Pediatr Ophthalmol Strabismus 2014;51 Online:e44-7.

51. Sarici SU, Yurdakök M, Unal S. Acute gastric dilatation complicating the use of mydriatics in a preterm newborn. Pediatr Radiol 2001;31:581-3.

52. Lim DL, Batilando M, Rajadurai VS. Transient paralytic ileus following the use of cyclopentolate-phenylephrine eye drops during screening for retinopathy of prematurity. J Paediatr Child Health 2003;39:318-20.

53. Kim HJ, Choi JG, Kwak K-H. Bronchoconstriction following instillation of phenylephrine eye drops in premature infants with bronchopulmonary dysplasia: two cases report. Korean $\mathrm{J}$ Anesthesiol 2015;68:613-6. 